\title{
I MPLEMENTATION OF REAL TIME AUTOCORRELATION METHOD EMPLOYING THE DS87C550 MICROCONTROLLER
}

\author{
A. Jiménez-Cañas, C. Fernández-Castellanos, V. Castro-Castillo, A. Ruiz-Toledo \& A. Sotomayor-Ortega.
}

\author{
Instituto de Cibernética, Matemática y Física (ICIMAF) \\ Calle D \# 551 /C y D, Vedado, cp. 10400 Habana, Cuba \\ Tel: 832 0771, Fax: 8333373 \\ antonio@icmf.inf.cu
}

Received: December $15^{\text {th }}$ 2004. Accepted February 25th 2005

\section{ABSTRACT}

In this work, the implementation of Real Time Autocorrelation method proposed by Takeuchi and Hogaki, is exposed. The purpose is to obtain the frequency of the fetal cardiac beats, originated from a Fetal Detector, using a wide-benefits DS87C550 microcontroller. The value of frequency is showed in a LCD. The autocorrelation algorithm was simulated in a PC to know its dynamic behavior from selected signals. A criterion to carry out the recognition and the selection of the first conformed crest, was obtained. By means of this criterion, the period of the processed signal is determined. Consequently, an optimized and original algorithm was created and implemented in the microcontroller, where the value of the fetal cardiac frequency (given in pulses per minute, ppm.) is obtained each $1.2 \mathrm{sec}$. In that time, two calculated values of frequency are averaged.

\section{RESUMEN}

En este trabajo se expone la implementación del método de la Autocorrelación en Tiempo Real de Takeuchi y Hogaki, empleando el microcontrolador DS87C550 de amplias prestaciones, con el objetivo de obtener el valor de la frecuencia de los latidos cardíacos fetales y mostrarlos en un visualizador LCD, a partir de la señal Doppler proveniente de un Detector Fetal. Se simuló el algoritmo de autocorrelación en una PC para conocer su comportamiento dinámico, a partir de diferentes señales de entrada, obteniéndose finalmente un criterio para llevar a cabo el reconocimiento y la selección de la primera cresta conformada, mediante la cual se determina el valor del período de la señal procesada. Consecuentemente, se creó un algoritmo original optimizado, implementado en el microcontrolador, mediante el cual se obtiene el valor de la frecuencia cardíaco fetal en pulsos por minuto (ppm). Cada 1,2 seg., como resultado de la promediación de dos valores de frecuencia calculados durante ese intervalo de tiempo.

KEYWORDS: Real Time Autocorrelation, Fetal Detector, Doppler.

\section{INTRODUCTION}

Among the Ultrasonic techniques of medical diagnosis, there exist the denominated Doppler techniques which have a wide range of applications in different medical specialties, e.g. Obstetrics. 
Besides, the use of the well-known Continuous Doppler Ultrasound has a very diverse use and where one of its important applications is constituted by the detection of the fetal cardiac beat, which allows the examination of anomalies and atypical behaviours presented during the gestation process [1]. However, the signals that originate from a fetal detector (FD) need to be processed to extract different information of interest, such as Doppler ultrasound signal, which is caused by fetal heart movements giving rise to fetal pulsations. This Doppler technique becomes essential during the diagnosis process.

Consequently, after the simulation of Real Time Autocorrelation in a PC, according to Takeuchi and Hogaki [2], [3], some results were obtained. This procedure can be used to extract the value of the repetition frequency of periodic signals, contaminated with random or semi-random noise, as the signal originated in an Ultrasonic Doppler FD does. A time-dependent waveform was selected, provid ing a correlation curve that facilitates the extraction of basic parameters to establish the implementation of the method. Also, a procedure was elaborated to select the value of the repetition period from the first crest of conformed autocorrelation function.

The results of the previously exposed simulation were implemented in a microcontroller of great benefit like the DS87C550 of Dallas-Maxim, having obtained satisfactory results. The proposed solution in the implementation of the method is totally original and parallelly, this processing method maintains its present use in systems and equipment conceived and designed for the same purpose.

\section{BASIC ELEMENTS}

Among the applications of autocorrelation, one of the most important is related to the identification of period in a periodic signal, which is contaminated with noise or some other signal of random characteristics [4]. The noise does not contribute with any periodic component; therefore, its autocorrelation does not produce maxima of any type. Therefore, the characteristics of a periodic signal are obtained independently from their magnitude, waveform and initial phase, being able to exist in signals with more than one periodic component, which originates secondary maxima.

Real Time Autocorrelation establishes the relation of a signal with itself in time, so the correlator model proposed by Takeuchi and Hogaki [2], [3] was used, according to fig 1. In this figure, observe that the first multiplier multiplies the word (16 bit), that is recirculating in a certain moment, given by the word registry, with the data in the input register, whose product $\mathrm{P}(\mathrm{n}, \mathrm{k})$, corresponds to kth round of nth period of recirculation, given by:

$$
P_{(n, k)}=D_{n} \times D_{(n-k)}
$$

Where:

$D(n-k) \leftarrow$ sampled data in $(n-k)$ th period of recirculation.

$D(n) \leftarrow$ sampled data in ( $n)$ th period of recirculation.

$\forall k<n$

Then, if the products $P(n, k)$ are added in a margin of $n$ values for each $k$, it would be obtained a value of autocorrelation by each value of $k$. Therefore, if all $P(n, k)$ values were stored during a typical period, it would be needed a large amount of memory for results warehousing. Luckily, this situation is solved using the method known as Exponential Mode Integration. 


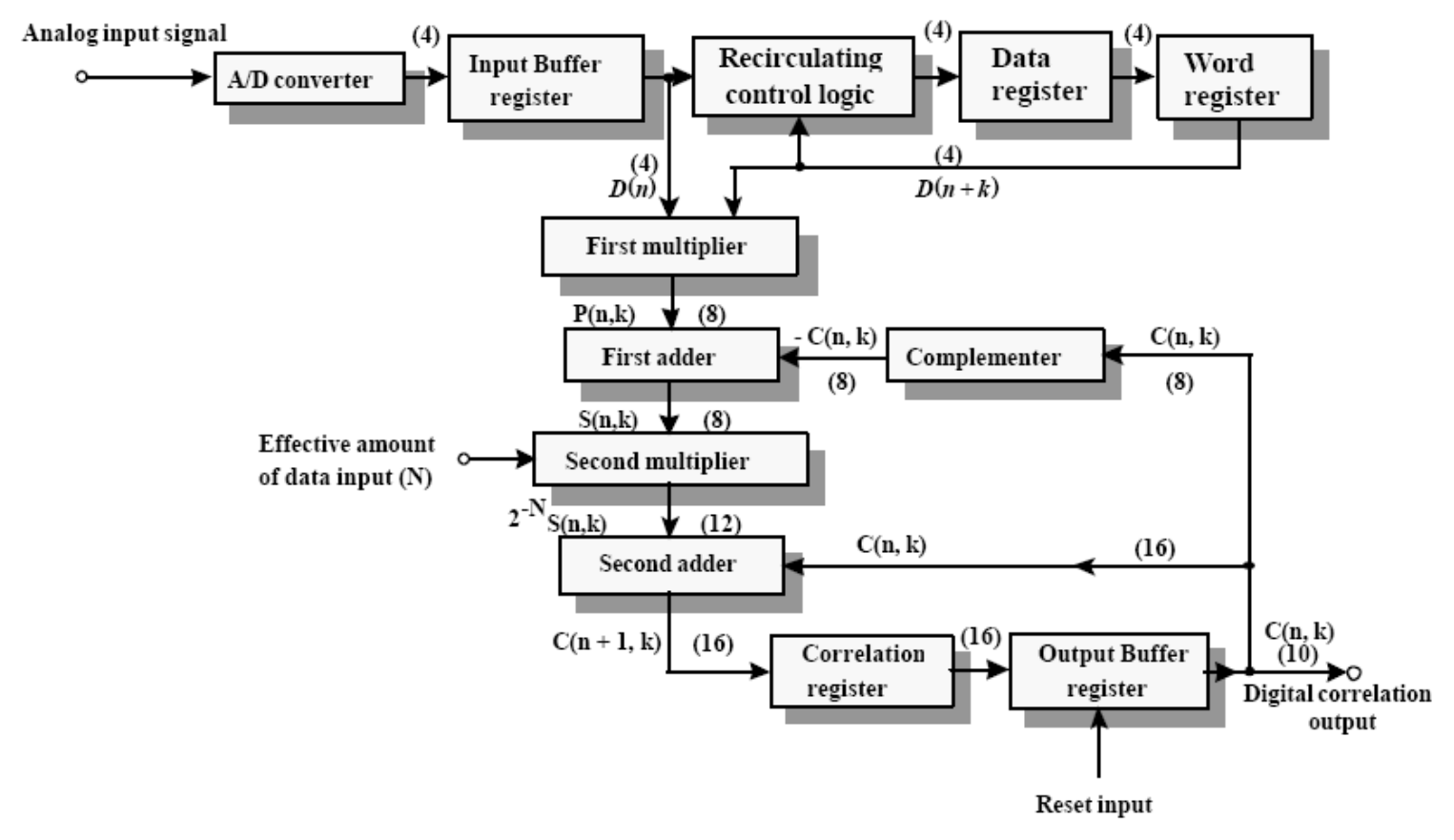

Figure 1. Block diagram of the algorithm proposed by Takeuchi and Hogaki

\section{EXPONENTIAL MODE INTEGRATION}

In agreement with figure 1, the output of the first multiplier and the value of previous autocorrelation, are applied to the first adder. The output of this adder is expressed:

$$
S_{(n, k)}=P_{(n, k)}-C_{(n, k)}
$$

where:

$C(n, k) \leftarrow$ autocorrelation.

$S(n, k) \leftarrow$ intermediate sum

Now, this intermediate sum $S(n, k)$ is applied to a second multiplier, which will be multiplied by the constant $\frac{1}{2^{N}}$

Parameter $\mathrm{N}$ is controlled externally according to the criterion shown in table I. 
Table I

\begin{tabular}{|cc|}
\hline FETAL FREQUENCY (PPM) & N \\
\hline$>160$ & 6 \\
\hline $80-160$ & 7 \\
\hline$<80$ & 8 \\
\hline
\end{tabular}

Next, the exit of the second multiplier and the value of previous autocorrelation are added in the second adder, whose sum replaces the previous value of autocorrelation. It must stand out that the operations carried out in the correlation register, are executed in synchronous way with respect to the operations that happen in the data register.

As a result, the correlation register will contain values of autocorrelation that depend in a certain way on the values previously calculated.

$$
\begin{gathered}
C_{(n+1, k)}=C_{(n, k)}+\frac{1}{2^{N}}\left\{P_{(n, k)}-C_{(n, k)}\right\} \\
C_{(n+1, k)}=\left\{1-\frac{1}{2^{N}}\right\} C_{(n, k)}+\frac{1}{2^{N}}\left\{D_{(n)} x D_{(n-k)}\right\}
\end{gathered}
$$

As a consequence, the rapidity of replacement of the values of autocorrelation will depend on the value of $\mathrm{N}$, that is, for large $\mathrm{N}$ values, the values of autocorrelation, which are in the correlation register, will depend to a lesser extent on the new sampled values and vice versa.

On the other hand, the behavio $r$ in time of this form of replacement or change of the autocorrelation values is similar to a capacitor-resistor network, which can be represented as an exponential function. Evaluating expression $3 \mathrm{~b}$ for a large number of terms yields the following expression:

$$
C_{(n, k)}=\frac{1}{2^{N}} \sum_{m=0}^{\infty}\left(1-\frac{1}{2^{N}}\right)^{m} x\left\{D_{(n-m)} x D_{(n-m-k)}\right\}
$$

where:

$$
\begin{gathered}
\left(1-\frac{1}{2^{N}}\right)^{m} \quad \leftarrow \text { represents the digital expression of an exponential function, and } \\
m \quad \leftarrow \text { represents quantity of samples. }
\end{gathered}
$$

Equation 4 corresponds to an ana logous mathematical expression that defines an exponentially weighted autocorrelation function, known as "Fano's Fast Autocorrelation Function" [5], 


$$
F_{\left(t_{0}, \tau\right)}=\frac{1}{\alpha} \int_{t=t_{0}}^{-\infty} \exp \left\{-\frac{t}{\alpha}\right\} x f(t) x f(t-\tau) x d t
$$

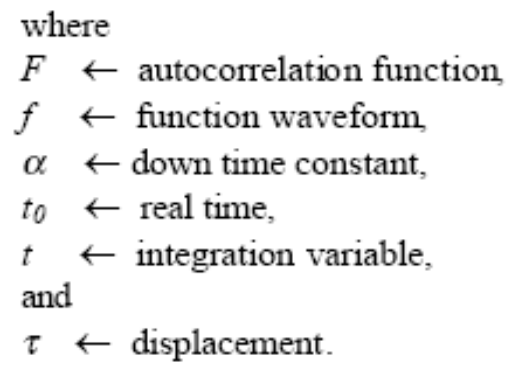

Fano's fast autocorrelation function is interpreted as the weighed average over the past time of the autocorrelation function, until an instant t0. The weight function $\frac{1}{\alpha} \exp ^{\left(\frac{t}{\alpha}\right)}$ corresponds with the impulse response of a RC low pass network.

From expression 4 , it is inferred that the effective amount of data $\mathrm{m}_{\mathrm{ea}}$, necessary to update the autocorrelation curve in a given time $T_{\text {ea, }}$ is determined exclusively for $\mathrm{N}$ or a values. Therefore, equalling the continuous and discrete exponential terms, and setting $\quad \mathrm{t}=\mathrm{a}$, the following considerations are taken into account

where, from 4 y 5 ;

$$
t=\alpha=T_{\mathrm{eq}}=m_{\mathrm{eq}} \times(\text { sampling time })
$$

$$
\forall: \quad \begin{aligned}
& \left(1-\frac{1}{2^{N}}\right)^{m_{e q}}=\exp ^{-1} \\
& m_{e q}=-\left[\ln \left(1-\frac{1}{2^{N}}\right)\right]^{-1}
\end{aligned}
$$

If $\mathrm{N} \gg 2$, then

or:

$$
m_{e q} \sim 2^{\mathrm{N}}
$$

$$
N^{\sim} \log _{2} m_{e q}
$$

Then, for a sampling time of 5 msec (200 Hertz), some values of meq and Teq are obtained according to table II:

Table //

\begin{tabular}{|c|c|c|}
\hline $\mathrm{N}$ & MEQ & TEQ \\
\hline 6 & 63,49 & $317,5 \mathrm{msec}$. \\
\hline 7 & 127,49 & $637,5 \mathrm{msec}$. \\
\hline 8 & 255,49 & $1,27 \mathrm{sec}$. \\
\hline
\end{tabular}

Therefore, taking into account the approximated margin of values that takes the fetal frequency, included from 60 to 210 ppm., whose periods oscillate from 1000 to $283 \mathrm{msec}$. respectively, $\mathrm{N}$ can be selected in a margin from 6 to 8 , according to the indicated intervals of fetal frequency in table 2, constituting this value of $N$ the reason of multiplication applied to the block named "second multiplier", Fig 1. It means that it is only necessary to wait for a time 
Teq to obtain the autocorrelation curve updated, using meq samples, according to the value of fetal frequency that is being measured.

\section{RESULTS OF SIMULATION}

Simulation of Real Time Autocorrelation, according to the model of Takeuchi and Hogaki, represented in equation 4, was implemented in a PC. The data of the pre-processed fetal signal were obtained by means of a PC-LAB acquisition card and a set of fetal signals, previously recorded in cassettes. The autocorrelation curve was composed of 250 points, assuming a data sampling period of 5 msec.

As a result of using several autocorrelation curves, starting from the previously mentioned data files, and other data files that contained values of known functions, some important experiences were obtained:

The behavior and the form of autocorrelation curve depend directly on the signal waveform to be correlated and the value of $\mathrm{N}$.

- Signals that do not have negative values present greater horizontal deviations with respect to those having negative values.

- Sinusoidal signals present greater displacements with respect to other signals.

- The low frequency signals present greater deviations than those of high frequency.

- The rectangular signals do not present deviation in horizontal direction.
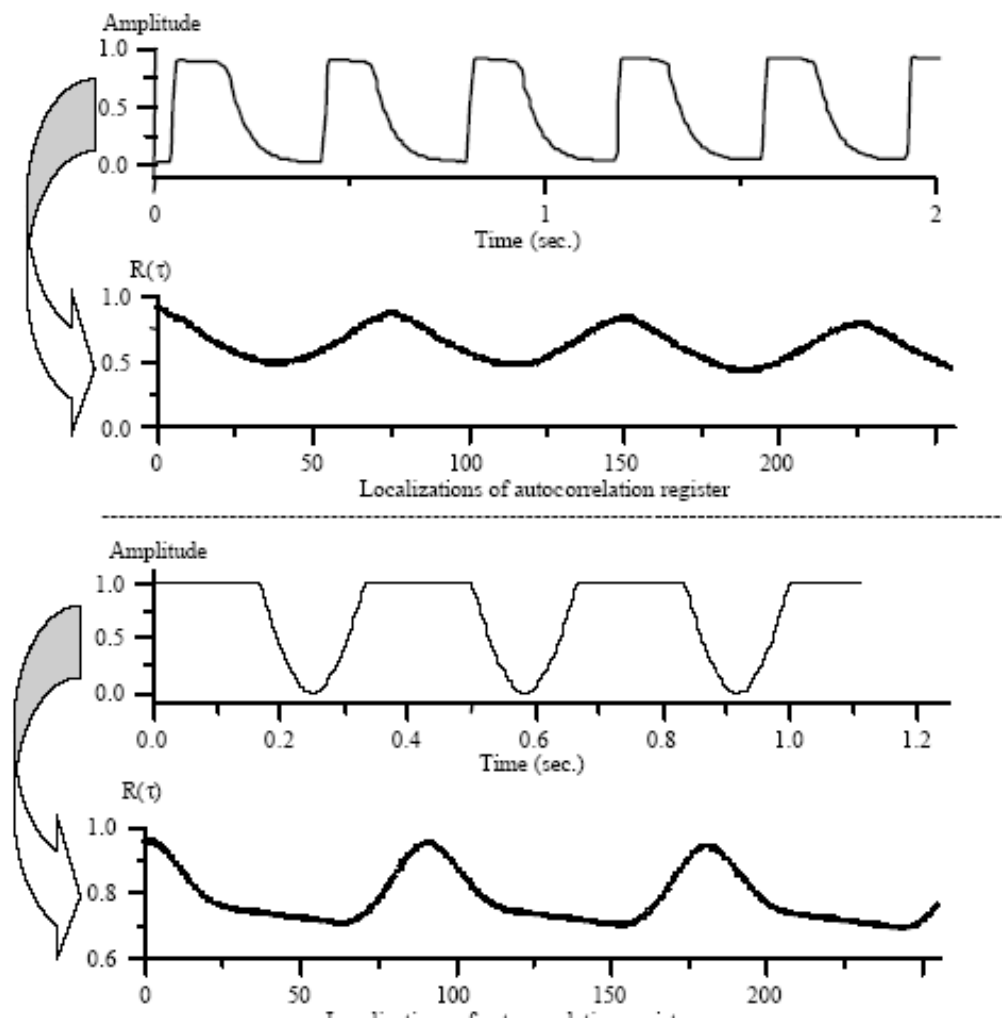

Localizations of autocorrelation register

Figure 2. Sample of the conformed selected signals and their curves of simulated autocorrelation 
- In general, smaller value s of $\mathrm{N}$ correspond to greater lateral deviations, diminishing its effect in rectangular signals.

Taking into account these aspects, some tests for $\mathrm{N}=8$ and different waveforms were carried out, giving rise to the conclusion that the best behavior obtained combines rectangular and sinusoidal waveforms. Finally, a trimmed sinusoidal waveform was selected, which combines good behavior of the rectangular signal and the characteristics of the sinusoidal signal, obtaining acceptable results with low horizontal displacement of the autocorrelation curve. However, the use of a rectangular signal allows circuit simplifications in general. In figure 2, two Doppler fetal signal waveforms appear, conformed to the criterion previously exposed.

\section{IDENTIFICATION OF THE FIRST MAXIMUM}

Once obtained the autocorrelation curve, it becomes necessary to identify absolute maximum values to know the behavior of them with time. For this, the 250 points of autocorrela tion are divided in five groups of 50 lines each one, with the objective to eliminate the possible ambiguity during the identification of the absolute maximum. As a result, a single absolute maximum within each group is allowed. This happens because 50 sampled lines with intervals of 5 milliseconds gives 250 milliseconds, which represents a fetal frequency of 240 ppm., that is outside the margin of values of interest (60-210 ppm.).

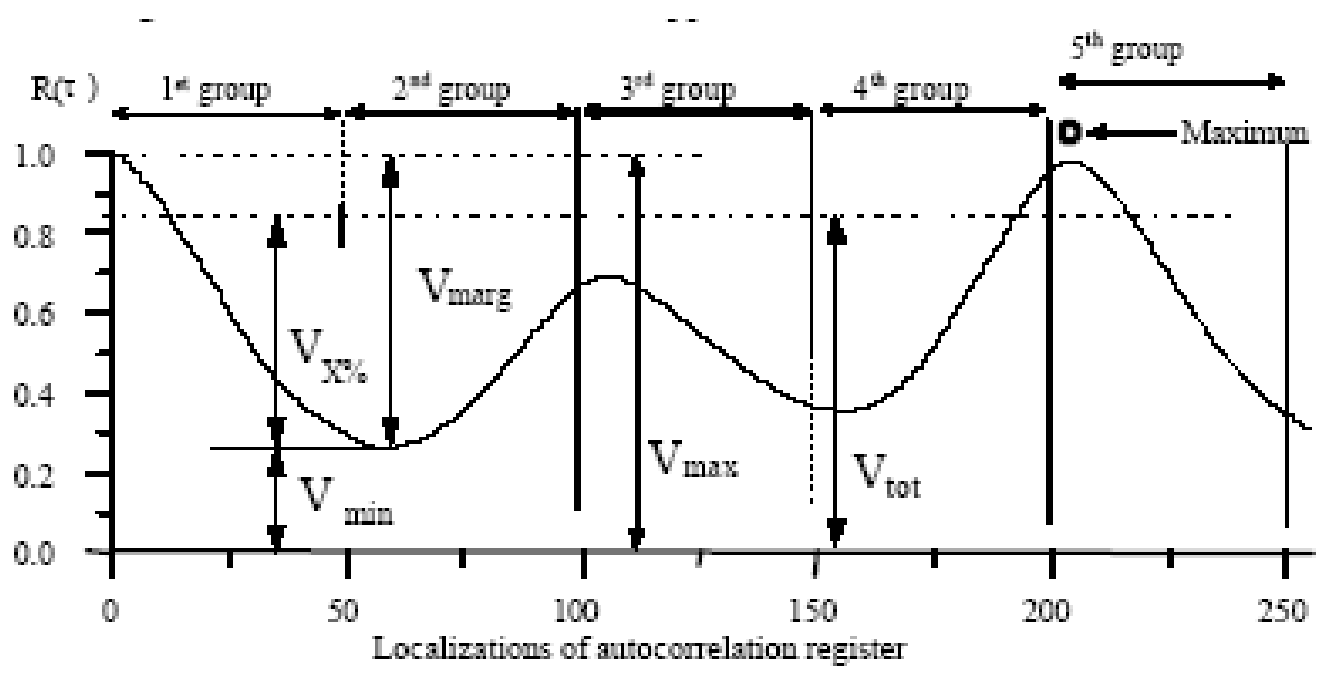

Figure 3. Indication of Vtot, Vmarg, $1 \times \%$, and Vmin for the determination of the first absolute maximum of autocorrela tion curve

According to figure 3, the highest and the smallest values of 250 calculated points are selected, represented by Vmax and $V_{\text {min }}$ respectively. Besides, the dynamic margin $V_{\text {marg }}$ is defined as the difference between $V_{\max }$ and $V_{\min }$. Then, a percent of the value $V_{\text {marg, }}$ represented by $V_{\mathbf{X} \%}$, is calculated and added to the minimum absolute value Vmin, obtaining the so called total value Vtot, that represents the selected value to carry out the comparisons. Now, the absolute maxima of each group of 50 values are selected and compared with respect to the total value Vtot. If absolute maxima values are greater than Vtot, they will be indicated during simulation by means of a dark point placed upon them. 


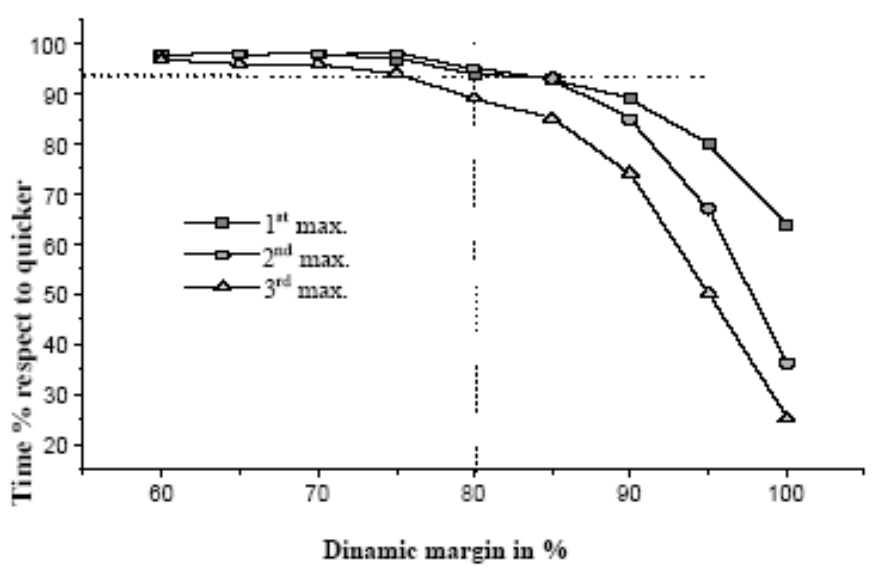

Figure 4. The selection of $V_{X \%}$ as $V_{80 \%}$ represents a compromise between speed of identificationof first maximum and overlapping with respect to the secondary maximum, during the process of signal period selection

The criterion for selection of $80 \%$ is based on the obtained results of the observations conducted during the simulation. Consequently, it was selected $\mathrm{V} \times \mathrm{x}$ as $\mathrm{V}_{80} \%$, since $80 \%$ of Vmarg provides a good and fast identification of the first absolute maximum with minimum influence of the secondary maximum, according to figure 4 . However, the greatest of all absolute maximum, being over $V_{80} \%$, is chosen in hardware implementation.

\section{OTHER RESULTS}

- A sampling frequency of 200 Hertz was selected (a sample each 5 msec.), as a commitment value between speed of processing and amount of points in the autocorrelation curve.

- From accumulated experience in algorithm simulation of fetal signals, it was reached the conclusion that for $\mathrm{N}=8$, in general, good stability is obtained with low horizontal displacement of the autocorrela tion curve.

- Because of the sampling period determines the resolution of the system, the accuracy of obtaining the values of the fetal frequency will depend on the values of signal frequency and sampling period. Therefore, there exists a reading error. with respect to the true value of period $T$ of the signal, given by $k T$, where $k$ depends on the two previously mentioned aspects. In figure 5 it is shown how the obtained exactitude varies nonlinearly respect to the values of fetal frequency. It is observed that, from 60 to $117 \mathrm{ppm}$., the value of the introduced error is null, from 115 to 155 ppm., it differs in 1 or 2 units, from 155 to 195 ppm., the error value is 3, and over 195, its magnitude might reach 3 units.

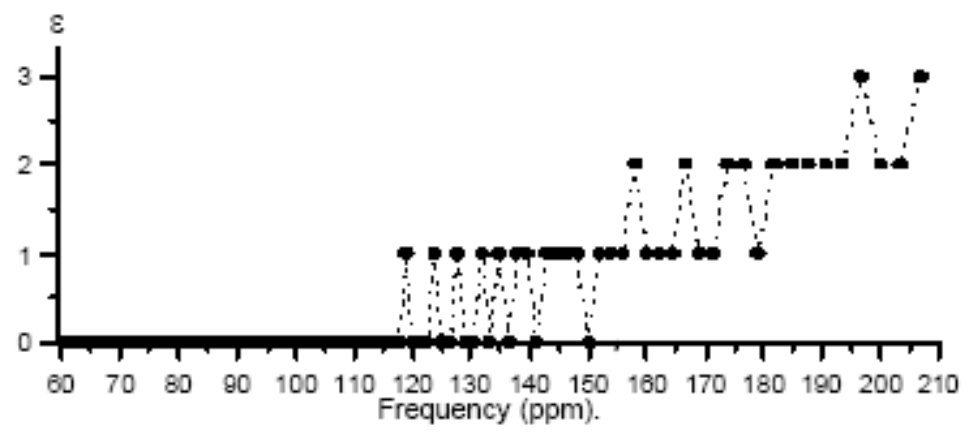

Figure 5. Nonlinear behaviour of committed error in the selection of signal discreet values versus frequency of beats, for a sampling frequency of $200 \mathrm{~Hz}$. The maximum value of reached error is 3 


\section{HARDWARE IMPLEMENTATION}

The basic diagram of implemented hardware is shown in fig 6, which is basically compound of a DF, a pre-processing section and a processing section. The processing section is constituted by a Dallas-Maxim DS87C550 microcontroller, decoders and a LCD visualizer

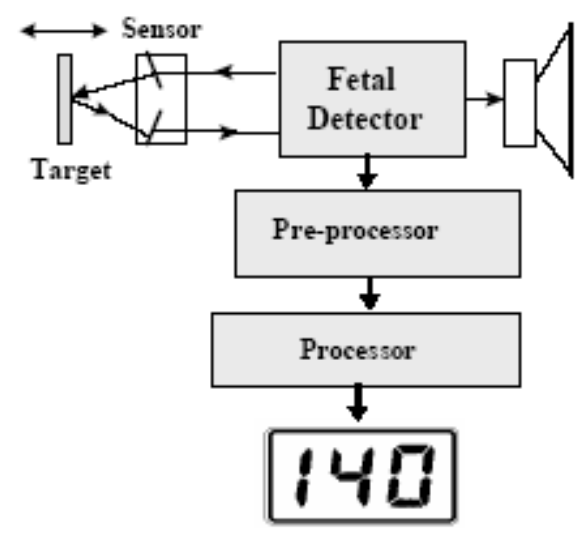

Figure 6. Block diagram of hardware

\section{FETAL DETECTOR}

It sends a $2 \mathrm{MHz}$. ultrasonic signal towards the fetal heart, and as a result, it receives an ultrasonic Doppler signal that carries the fetal cardiac information. Then, the ultrasonic signal is transformed into an electrical signal, which is amplified, filtered and amplitude-detected, obtaining finally an audible Doppler signal (bounded between 100 and $900 \mathrm{~Hz}$ ), whose sound is proportional to the movements that occur in the fetal heart, including the ones caused by the blood flow, whose average spectral composition is shown in figure 7. This audible signal presents the aspect of an amplitude-modulated signal, whose "modulating envelope" has a pulsating and periodic waveform, in correspondence with the fetal heart rate.

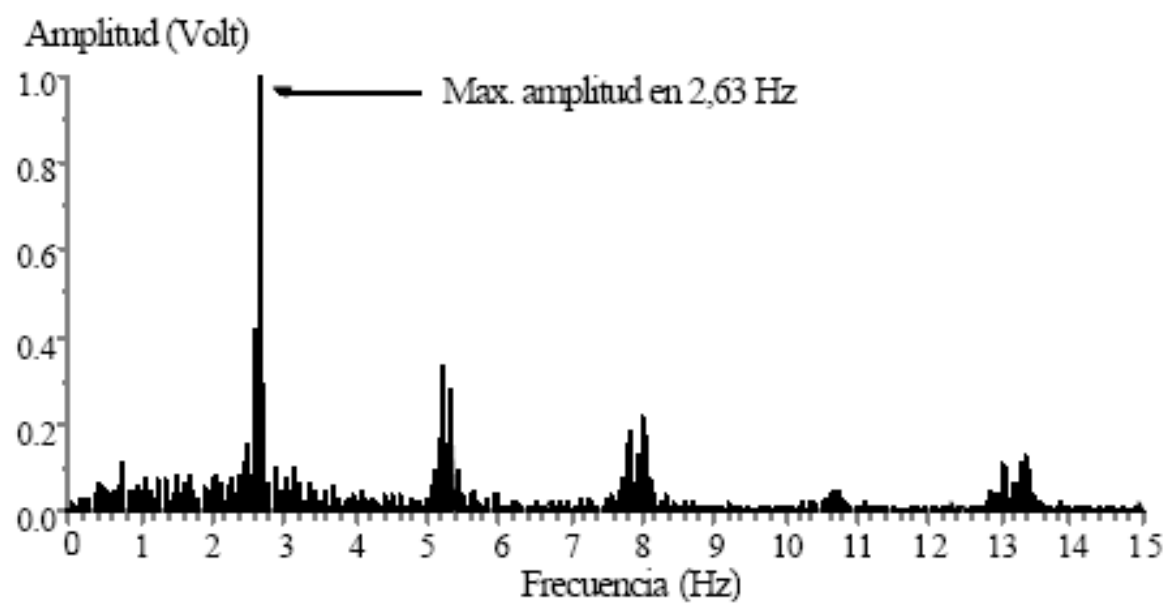

Figure 7. Average spectral composition of a Doppler signal generated by a FD 


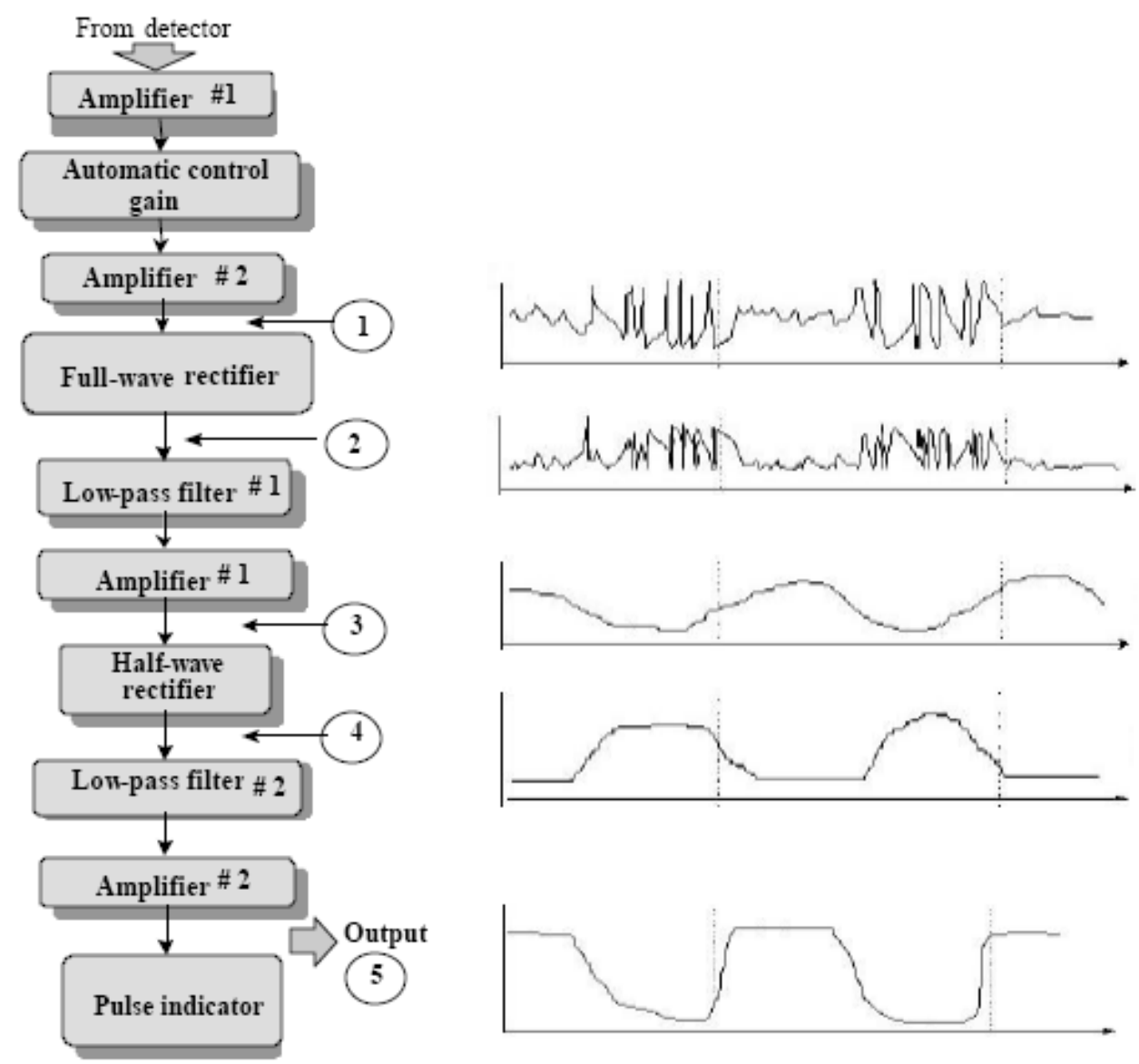

Figura 8. Block diagram of the pre-processor showing waveforms that appear in different sections

\section{PRE-PROCESSING}

The "modulating envelope" contains the fetal cardiac information, whose frequency repetition range is included from 0.83 to $3.5 \mathrm{~Hz}$ (50-210 ppm). Groups of several complete cycles of the audible signal, 5 to 30 cycles approximately, compose their envelopes. If amplitude demodulation is applied to this signal, the components of smaller frequency, contained in the envelope, are transferred to baseband, obtaining the fetal cardiac data or the corresponding one to the pulsations, its harmonic components and some noise. Therefore, if a suitable low-pass filtering is applied, the purity of smaller frequency signals can be improved. Figure 8 shows these aspects.

However, the pre-processing does not eliminate completely random variations that modify to a certain extent the filtered signal. Therefore, it is required the application of a processing method that allows obtaining the data of the fetal cardiac frequency in a continuous and reliable way, that is, the pre-adapted filtered signal facilitates the procedure to obtain a suitable curve of real time autocorrelation by means of a microcontroller.

\section{PROCESSING}

A sampling frequency of $200 \mathrm{~Hz}$ (a sample each $5 \mathrm{msec}$.) was selected as a commitment value between speed of processing in the microcontroller, and amount of points in the autocorrelation curve. The algorithm that calculates the autocorrelation was optimized to the maximum and as a result, it could be used $6 \mathrm{MHz}$ as frequency of microcontroller's clock. On the other hand, the possibility of selecting three values of $\mathrm{N}$ in the implementation of the 
algorithm, reduces the margin of possible frequencies to measure, increasing the duration of the autocorrelation circular subroutine.

Besides, from the results obtained in the algorithm simulation with fetal signals, it was reached the conclusion that selecting to a value of $N=8$, good stability is obtained with respect to the horizontal displacement of the autocorrelation curve. Therefore, fixing the value of $\mathrm{N}$, the size and the duration of the assembly subroutine are reduced, which calculates the circular autocorrelation in only $33 \mathrm{msec}$. (see figure 9) and finally, allows to carry out 240 rounds of autocorrelation with 150 values per round, for $\mathrm{N}=8$. In this way, good results as exactitude and stability of reading are obtained in all the margin of measurement, from 60 to $210 \mathrm{ppm}$.

Besides, these 240 rounds are equivalent to a processing time of $1.2 \mathrm{sec}$., which supposes a meq of 240, according to previously said, corresponding a va lue of $\mathrm{N}$ between 7 and 8 . Then every 1.21 seconds approximately, the display is refreshed with a new value of reading, being it, an acceptable time according to the minimum value of measured fetal frequency of $60 \mathrm{ppm}$, whose period is 1 second. Therefore, using an $N=8$, it gives sufficient cover so that the autocorrelation curve is updated in this time. Finally, the 240 rounds are divided in two sections of 120, with the purpose to obtain an average value that improve the interpolation of frequency values in the reading, mainly, at the moments at which the fetal frequency changes slightly of value.

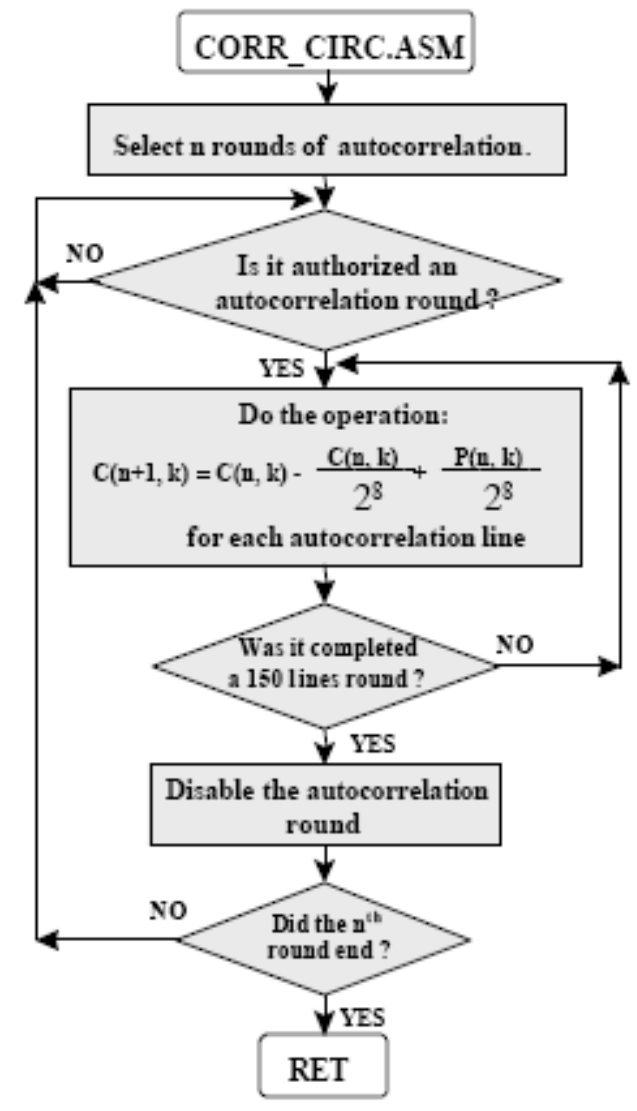

Figure 9. Block diagram of the circular correlation subroutine

On the other hand, Maxim-Dallas DS87C550 microcontroller is a high speed new generation device which is 8751 compatible, including the instructions set and compiler [6]. It is 2.5 times quicker than 8751 microcontroller for equal clock frequencies, that is, if it is operating to lower frequencies, the same performance in equal time interval can be obtained with a lower current consumption. 


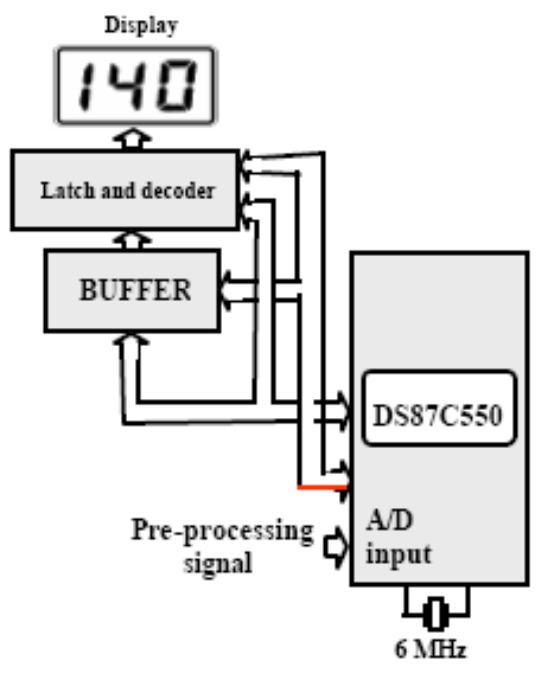

Figure 10. Block diagram of implemented hardware using the DS87C550

It can operate up to $33 \mathrm{MHz}$, which allows the speed of an elementary autocorrelation round to be increased in four times. In addition, it has an 8 Kbytes, reconfigurable ROM memory, A/D converter with a conversion time of 20 msec. and several working modes that facilitate the lowcurrent consumption. Figure 10 shows the implemented architecture and figure 11 shows two photos of hardware implementation.
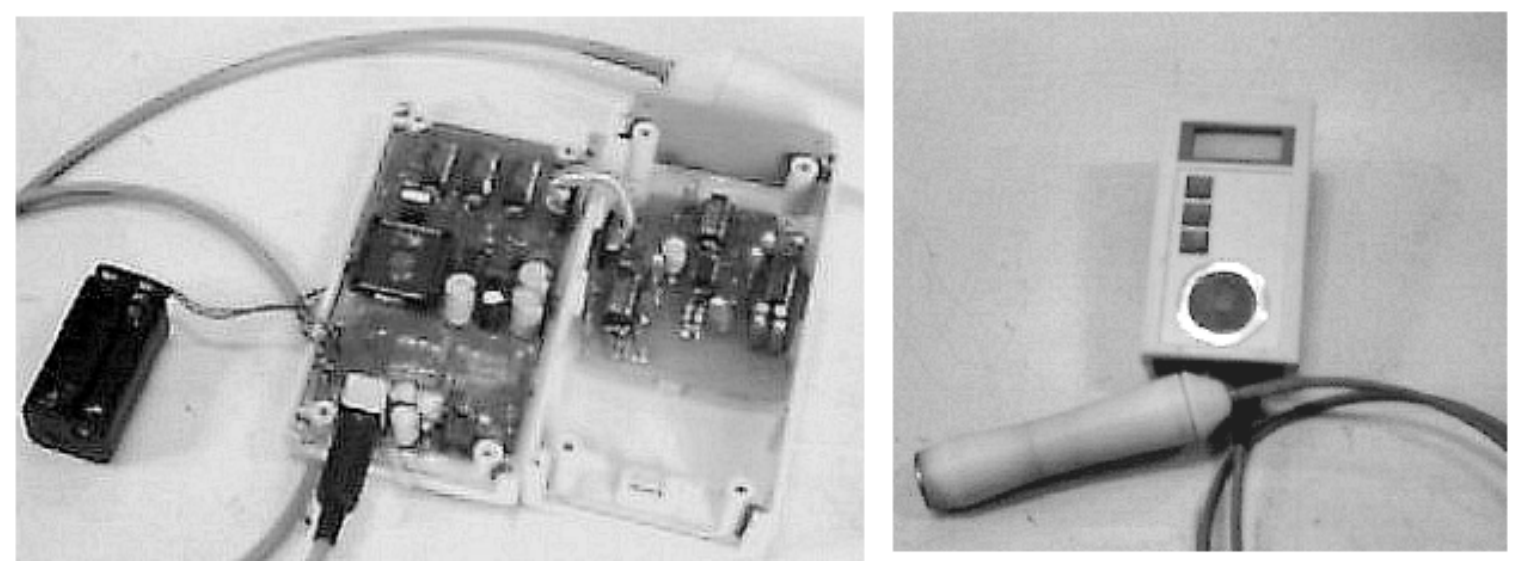

Figure 11. Internal and external view of the implementation

\section{FEATURE ADVANTAGES}

- It simplifies the architecture of the processing stage with the presence of A/D converter and ram internal memories, which aid to reduce cost and volume (see figure 10).

- It runs the 8051 software with an averaged increment in speed of 2.12, which allows diminishing the frequency of the clock and therefore the consumption of current.

- Greater facility in device handling and the implementation of new possibilities of operation, since it has 6 bidirectional ports and six- level external interrupts.

- Its mechanisms facilitate the auto-shutoff.

- The low consumption working modes contribute to portability and the use of batteries. 


\section{FINAL CONSIDERATIONS}

- The pre-processing stage can be optimized using low consumption devices and suitable circuital structures.

- The used processing method is up to date in commercial equipments due to the simplicity of its implementation.

- The design is left open to the use of other devices and methods, even in pre-processing and processing.

\section{REFERENCES}

[1] Botella, J. \& Clavero, J. “Tratado de Ginecología”, tomo 1, Editorial Científico Técnica, 1983.

[2] Takeuchi Y. "U.S. patent 3991365 (9 Nov. 1976)".

[3] Takeuchi, Y. \& Hogaki, M. "An Adaptive Correlation Ratemer: A New Method for Doppler Fetal Rate Measurements", Ultrasonics, may 1978.

[4] Proakis J.G. \& Manolakis D.G., "Digital Signal Processing: Principles, Algorithms and Application", Mac Milland Publishing Co., New York 1992.

[5] Fano, R. "Short-Time Autocorrelation Function and Power Spectra", J.A.S.A. Vol. 22, No. 5, september 1950.

[6] Dallas-Maxim Semiconductors, "High Speed Microcontroller User's Guide", Maxim Integrated Products, 2004. 